\title{
Correspondence
}

\section{Optimized Nonuniform Constellation Rearrangement for Cooperative Relaying}

\author{
Akram Bin Sediq, Petar Djukic, Halim Yanikomeroglu, and
} Jietao Zhang

\begin{abstract}
The performance of cooperative relaying networks can be significantly enhanced by using constellation rearrangement (CoRe). In CoRe, the base-station and the relay-station use different constellations, each having the same number of signal points, to communicate with the user terminal. A number of CoRe schemes have been proposed in the literature based on uniform quadrature-amplitude modulation (QAM) constellations. However, it is still unclear whether nonuniform QAM constellations can further enhance the performance of CoRe. Toward this end, we investigate the problem of designing the optimum nonuniform QAM constellations for CoRe. Our motivation is that nonuniform constellations have the potential to outperform their uniform counterparts because the set of nonuniform constellations is a superset of uniform constellations. Nonuniform QAM constellations can be categorized as either decomposable or nondecomposable. Unlike nondecomposable QAM constellations, decomposable QAM constellations are generated from the Cartesian product of two pulse-amplitude modulation (PAM) constellations. We formulate an optimization problem to find the nonuniform constellations that have the minimum union bound on the uncoded symbol error rate (SER). Using convex analysis, we devise a search method to find globally optimum nonuniform decomposable constellations. We also devise a simple heuristic to find good locally optimum nonuniform nondecomposable constellations, which perform better than their decomposable counterparts.
\end{abstract}

Index Terms-Convex optimization, cooperative relay networks, nonuniform constellation rearrangement (CoRe).

\section{INTRODUCTION}

Cooperative relaying is widely accepted as an enabling technology that is designed to meet the increasing demand for ubiquitous highdata-rate coverage. In cooperative relaying, the signal reaches its destination in multiple hops through intermediate relays. Instead of solely relying on the signal from the last hop, the receiver terminal properly combines all signals from the intermediate hops [3]. The performance of cooperative relaying networks can be further enhanced by using constellation rearrangement (CoRe) [4]-[6], which is a tech-

Manuscript received August 24, 2010; revised November 20, 2010 and February 11, 2011; accepted March 10, 2011. Date of publication April 21, 2011; date of current version June 20, 2011. This work was supported in part by Huawei Wireless Research, China, under Grant YBWL2008087 and in part by an Ontario Graduate Scholarship. This paper was presented in part at the 25th Queen's Biennial Symposium on Communications, Kingston, ON, Canada, May 2010, and the IEEE 71st Vehicular Technology Conference, Taipei, Taiwan, May 2010. The review of this paper was coordinated by Prof. N. Arumugam.

A. Bin Sediq and H. Yanikomeroglu are with the Department of Systems and Computer Engineering, Carleton University, Ottawa, ON K1S 5B6, Canada (e-mail: akram@sce.carleton.ca; halim@sce.carleton.ca).

P. Djukic was with the Department of Systems and Computer Engineering, Carleton University, Ottawa, ON K1S 5B6, Canada. He is now with the Communications Research Centre Canada, Ottawa, ON K2H 8S2, Canada (e-mail: petar.djukic@crc.gc.ca).

J. Zhang is with the Communications Technologies Research Department, Central Research and Development Unit, Huawei Technologies Co., Ltd., Shenzhen 518129, China (e-mail: jtzhang@ @uawei.com).

Color versions of one or more of the figures in this paper are available online at http://ieeexplore.iee.org.

Digital Object Identifier 10.1109/TVT.2011.2145013
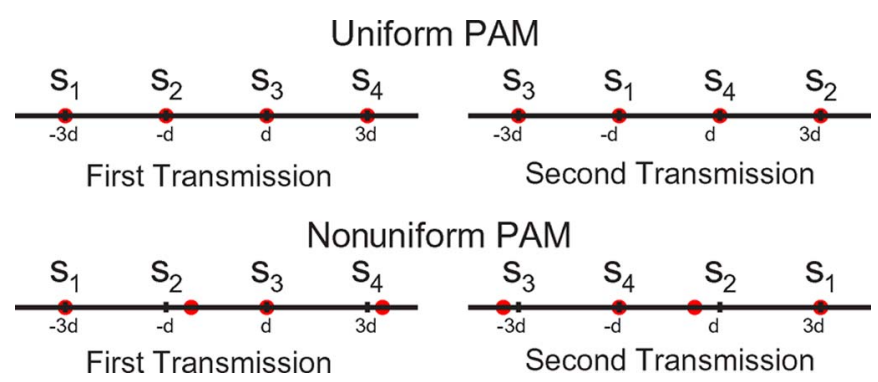

Fig. 1. Uniform and nonuniform CoRe (4-PAM).

nique that uses different constellations for different retransmissions without changing the modulation level. In $\mathrm{CoRe}$, the source uses a constellation that is different from that used by the relay, and both constellations have the same number of constellation points. This technique is also called trans-modulation [4], constellation change [5], and symbol mapping diversity [7], but in this paper, we use the term "CoRe." The key question in designing CoRe is to find the optimum constellations for two (or more) transmissions.

There are multiple ways to find constellations for CoRe. One way is to keep the same constellation in both transmissions but to remap the symbols in the constellations. For example, in Fig. 1, we show two uniform pulse-amplitude modulation (PAM) constellations that are different only in their assignment of symbols to constellation points. The constellation points are uniformly spaced on the real line; thus, we call these types of constellations uniform constellations.

Remapping symbols for different transmissions was originally proposed for hybrid automatic repeat request (HARQ) with soft combining [8], [9]. In the HARQ context, if the destination receives an erroneous packet, it asks the source for a retransmission and keeps the erroneous packet to combine it with the retransmitted packet. Since combining transmissions received at different times is a common feature in both HARQ and cooperative relaying, CoRe was also proposed for cooperative relaying applications [4]-[6]. To the best of our knowledge, all of the existing CoRe schemes remap uniform PAM or uniform quadrature-amplitude modulation (QAM) constellations.

Despite the progress made in CoRe, it is still unclear whether nonuniform constellations can further increase the gain achieved by CoRe. Toward this end, we study the problem of finding the optimum nonuniform CoRe. In the case of nonuniform PAM constellations, the symbols are allowed to be anywhere on the real axis while taking energy restrictions into account (see Fig. 1). Allowing free floating of symbols adds another dimension to the problem, beyond what is currently in the literature. Finding the optimum nonuniform CoRe not only requires optimizing the symbol mappings for different transmissions but also requires optimizing the constellations themselves. Our motivation is that nonuniform constellations have the potential to outperform their uniform counterparts because the set of nonuniform constellations is a superset of uniform constellations.

The idea of using nonuniform constellations has been proposed for applications other than CoRe. For example, in [10], the authors use nonuniform signal constellations in ARQ (without combining transmissions at the receiver or changing the signal constellation at the transmitter) to adapt the data rate to the received signal-to-noise ratio (SNR) in a fading channel, without using channel state feedback 


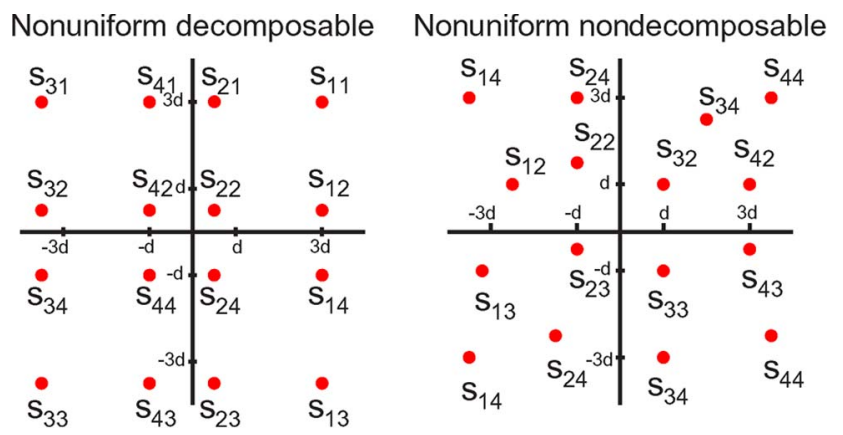

Fig. 2. Nonuniform decomposable and nondecomposable constellations (16-QAM).

or incremental redundancy. As far as we know, the use of nonuniform constellations for CoRe has not yet been investigated.

We further categorize QAM constellations into decomposable and nondecomposable constellations. Decomposable QAM constellations are those generated from the Cartesian product of two real PAM constellations. For example, the nonuniform decomposable 16-QAM constellation in Fig. 2 is the Cartesian product of the nonuniform 4-PAM constellation for the second transmission shown in Fig. 1 with itself. The real and imaginary parts of decomposable constellations are independent and can be separately decoded, which reduces decoding complexity.

Nondecomposable constellations are not necessarily constructed from the Cartesian product of two real PAM constellations. In Fig. 2, we show an example of a nonuniform nondecomposable 16-QAM constellation. Nondecomposable constellations need a more complicated decoder than decomposable constellations. However, as we will show later, the gains achieved by using nondecomposable constellations may well be worth the additional complexity.

We start by formulating the optimization problem to find the optimum nonuniform constellations that minimize the union bound on the uncoded SER. Using convex analysis, we show that, in the case of nonuniform decomposable constellations, the space of constellations can be partitioned in a way that results in a finite number of convex optimization problems. Based on this observation, we devise a search method to find the best possible decomposable constellations over all the partitions.

For the case of nonuniform nondecomposable constellations, the optimization problem is not convex, meaning that we cannot find its global minimum efficiently. Nevertheless, the convex optimization techniques still allow us to find local minima, which can lead to good solutions, provided that the solver is given a good initial point. We use optimum nonuniform decomposable constellations as initial points for the solver; thus, the local minima obtained by the solver will be at least as good as the optimum nonuniform decomposable constellations.

\section{RELATED WORK}

In this Section, we give an overview of the key CoRe schemes that have been proposed in the literature. The first CoRe scheme was proposed for HARQ [8], [11]. The key idea in this CoRe scheme is to average the variations in bit reliabilities inherited in Gray-coded multilevel modulation schemes (such as PAM and QAM). This scheme was also proposed for cooperative relaying in [5].

In [9], the authors observed that the CoRe scheme proposed in [11] is designed in an "ad hoc" way without an optimization. By considering the modulation level and number of retransmissions as an augmented signal space, the authors proposed a CoRe scheme that maximizes the minimum distance in this augmented signal space. This
CoRe scheme is also extended for multiple retransmissions combined with space-time block coding in [12].

In [13], the authors proposed the use of uniform $M$-QAM constellations in the first transmission and the subsequent $\left(\left\lfloor\log _{2} M / 2\right\rfloor-1\right)$ retransmission(s) and the use of nonuniform constellation in the further retransmissions. The nonuniform constellation used is in effect a quadrature phase-shift keying (QPSK) constellation that carries 2 bits/ symbol, and the rest of the bits $\left(\log _{2} M-2\right)$ are not transmitted. Since conventional QAM and QPSK constellations are used, where the constellation points are evenly spaced, these constellations are "nonuniform" in a different sense than those described in this paper.

In [7], the authors assumed that the transmitter has full channel state information (CSI) and that it adapts its constellation according to the instantaneous SNR. The authors proposed their scheme for HARQ. They formulated the union bound as an upper bound for the bit error rate (BER), and they found the constellations that minimize this bound. However, having full CSI at the transmitter adds significant complexity due to the requirement for a fast feedback channel to report the CSI to the transmitter.

Since combining retransmissions is a common feature in both HARQ and cooperative relaying, CoRe was also proposed for cooperative relaying [4]-[6]. In [4], a CoRe scheme was proposed to improve the performance of cooperative relaying. The authors formulated a bound on the symbol error rate (SER), but they concluded that finding the constellations that minimize the bound is extremely complex, and they provided a heuristic solution.

In [6], the authors adopted an approach that is similar to [7] but for cooperative relaying without CSI at the transmitter. Using exhaustive search, the authors found the constellations that minimize the union bound on the uncoded BER in Rayleigh fading. Simulation results show that the CoRe scheme that was proposed in [6] outperforms the other existing CoRe schemes with comparable complexity.

It is worth repeating that uniform constellations are used in all existing CoRe schemes. In this paper, we extend the CoRe concept to nonuniform constellations.

\section{SYSTEM MODEL}

We consider a network that consists of a base station (BS), a relay station (RS), and a receiving user terminal (UT), each using a single antenna. The RS assists the UT, which suffers from poor channel conditions. The RS uses the decode-and-forward strategy-it fully decodes the signal it receives from the BS before reencoding and forwarding it to the UT.

The RS operates in half-duplex mode, on orthogonal channels. To facilitate the discussion, we assume that half-duplexing at the RS is achieved with time-division duplexing (TDD).

Transmitters use $M$-QAM constellations, where $M$ is the number of constellation points. Without any loss of generality, the $M$-QAM constellation has an average energy per symbol equal to unity. In the conventional setup, both the BS and the RS use the same constellations for all transmissions, whereas in CoRe, the BS and the RS use different constellations. We denote the signal constellation points used in the first transmission with $\left\{s_{1}^{(1)}, s_{2}^{(1)}, \ldots, s_{M}^{(1)}\right\}$ and the signal constellation points used in the second transmission with $\left\{s_{1}^{(2)}, s_{2}^{(2)}, \ldots, s_{M}^{(2)}\right\}$. For example, if the symbol $s_{3}^{(1)}$ is to be transmitted to the UT, $s_{3}^{(1)}$ will be transmitted in the first transmission, whereas $s_{3}^{(2)}$ will be transmitted in the second transmission. The constellation points, which are complex numbers, are denoted by

$$
s_{k}^{(1)} \triangleq x_{k}^{(1)}+j y_{k}^{(1)} \quad \text { and } \quad s_{k}^{(2)} \triangleq x_{k}^{(2)}+j y_{k}^{(2)}
$$

where $\jmath \triangleq \sqrt{-1}$. 
To simplify the discussion, the system model is abstracted to the generic case where the UT receives two copies of the same information symbol but using different constellations. A specific relaying protocol that fits this generic case is discussed in Section V-A.

In one frame, the signals received by the UT in the first and second time slots are given by

$$
r^{(1)}=\alpha^{(1)} s^{(1)}+z^{(1)} \text { and } r^{(2)}=\alpha^{(2)} s^{(2)}+z^{(2)}
$$

respectively, where $\alpha^{(1)}$ and $\alpha^{(2)}$ are the channel coefficients, capturing the effects of path loss and small-scale fading, and $z^{(1)}$ and $z^{(2)}$ are additive white Gaussian noise samples.

Channel coefficients $\alpha^{(1)}$ and $\alpha^{(2)}$ are assumed to be known at the receiver for coherent detection. Coefficients $\left|\alpha^{(1)}\right|$ and $\left|\alpha^{(2)}\right|$ are modeled as independent Rayleigh random variables. Noise samples $z^{(1)}$ and $z^{(2)}$ are independent and identically distributed circularly symmetric complex Gaussian random variables with zero mean and variance $N_{0} / 2$. The average SNRs in the first and second transmissions are $\bar{\gamma}^{(1)}=E\left[\left|\alpha^{(1)}\right|^{2}\right] / N_{0}$ and $\bar{\gamma}^{(2)}=E\left[\left|\alpha^{(2)}\right|^{2}\right] / N_{0}$, respectively.

The UT utilizes signals received from the two independent branches and achieves spatial diversity. Similar to [4], [6], and [9], a maximumlikelihood (ML) decoder is assumed at the UT, which reduces to a minimum-distance detector.

We consider decomposable and nondecomposable $M$-QAM constellations. Decomposable $M$-QAM constellations are constellations that are generated from the Cartesian product of two real $\sqrt{M}$-PAM constellations. In other words, $\log _{2} \sqrt{M}$ bits are encoded in the real part of the constellation, and the other $\log _{2} \sqrt{M}$ bits are encoded in the imaginary part of the constellation. As a result, the real and imaginary parts of the constellation points are independent and can be separately decoded, which reduces the complexity of the ML decoder. On the other hand, nondecomposable constellations are not necessarily constructed from the Cartesian product of two real $\sqrt{M}$ PAM constellations.

The ML decoder estimates the transmitted symbol from $r^{(1)}$ and $r^{(2)}$ as $\hat{s}=s_{\hat{i}}^{(1)}$, where

$$
\hat{i}=\underset{i=1, \ldots, M}{\arg \min }\left\{\left|r^{(1)}-\alpha^{(1)} s_{i}^{(1)}\right|^{2}+\left|r^{(2)}-\alpha^{(2)} s_{i}^{(2)}\right|^{2}\right\} .
$$

For a complex number $x, x^{*}$ is the conjugate of $x$, and $|x|^{2}=x^{*} x$. The previously described detector requires a total of $M$ computations to decode one symbol.

For the special case of decomposable constellations, decoding complexity is significantly simplified by decoding the real and imaginary parts separately, ${ }^{1}$ as $\hat{s}=x_{\hat{i}}^{(1)}+\jmath y_{\hat{j}}^{(1)}$, where

$$
\begin{aligned}
\hat{i}=\underset{i=1, \ldots, M}{\arg \min }\{ & \left(\mathcal{R}\left(\tilde{r}^{(1)}\right)-\left|\alpha^{(1)}\right| x_{i}^{(1)}\right)^{2} \\
& \left.+\left(\mathcal{R}\left(\tilde{r}^{(2)}\right)-\left|\alpha^{(2)}\right| x_{i}^{(2)}\right)^{2}\right\} \\
\hat{j}=\underset{i=1, \ldots, M}{\arg \min }\{ & \left(\mathcal{I}\left(\tilde{r}^{(1)}\right)-\left|\alpha^{(1)}\right| y_{i}^{(1)}\right)^{2} \\
+ & \left.\left(\mathcal{I}\left(\tilde{r}^{(2)}\right)-\left|\alpha^{(2)}\right| y_{i}^{(2)}\right)^{2}\right\}
\end{aligned}
$$

$\tilde{r}^{(1)}=e^{-\jmath \angle \alpha^{(1)}} r^{(1)}, \tilde{r}^{(2)}=e^{-\jmath \angle \alpha^{(2)}} r^{(2)}, \mathcal{R}(x)(\mathcal{I}(x))$ is the real (imaginary) part of a complex number $x$, and $\angle x$ is the angle of a

\footnotetext{
${ }^{1}$ We assume that the bits encoded in the real (imaginary) part by the BS are also encoded in the real (imaginary) part by the RS.
}

complex number $x$ in radians. Since the constellation in this case is formed by a Cartesian product of two $\sqrt{M}$-PAM constellations, each of the sets $\left\{x_{1}^{(t)}, \ldots, x_{M}^{(t)}\right\}$ and $\left\{y_{1}^{(t)}, \ldots, y_{M}^{(t)}\right\}$, for $t \in\{1,2\}$, consists of $\sqrt{M}$ distinct elements. Consequently, finding $\hat{i}$ (or $\hat{j}$ ) requires $\sqrt{M}$ computations, which means that the ML decoder for decomposable constellations requires only $2 \sqrt{M}$ computations per symbol instead of $M$ computations per symbol.

\section{Optimum NONUNIFORM CONSTELlation REARRANGEMENT}

We formulate the optimization problem that searches for the complex numbers corresponding to constellation points that have the minimum union bound on the uncoded SER. The optimization problem is given as

$$
\begin{array}{cc}
\underset{\substack{s_{i}^{(1)}, s_{i}^{(2)} \\
\forall i \in\{1, \ldots, M\}}}{\operatorname{minimize}} & \sum_{i=1}^{M} \sum_{j=i+1}^{M} \frac{2 C}{\left|s_{i}^{(1)}-s_{j}^{(1)}\right|^{2}\left|s_{i}^{(2)}-s_{j}^{(2)}\right|^{2}} \\
\text { subject to } & \frac{1}{M} \sum_{i=1}^{M}\left|s_{i}^{(t)}\right|^{2} \leq 1 \quad \forall t \\
& s_{i}^{(1)}, s_{i}^{(2)} \in \mathbb{C} \quad \forall i
\end{array}
$$

where $C=16\left(M \bar{\gamma}^{(1)} \bar{\gamma}^{(2)}\right)^{-1}$ is a constant related to SNRs; variables $s_{i}^{(1)}$ and $s_{i}^{(2)}$ (for $1 \leq i \leq M$ ) correspond to the constellation points used in the first and second transmissions, respectively; and $\mathbb{C}$ is the set of complex numbers. Objective function (3a) minimizes the SER bound ${ }^{2}$ and was directly derived by applying the union bound on the pairwise symbol error probability [6, eq. (6)]. ${ }^{3}$ Constraints (3b) limit the average energy per symbol in each transmission to unity. Constraint (3c) implies that the constellation points can take any values in the complex space, allowing nonuniform constellations. We tackle (3) for decomposable and nondecomposable constellations separately.

\section{A. Decomposable Constellations}

Since designing decomposable $M$-QAM constellations is equivalent to designing $\sqrt{M}$-PAM, we formulate the optimization problem for $\sqrt{M}$-PAM constellations. The optimum decomposable $M$-QAM constellation is then the Cartesian product of the optimum $\sqrt{M}$ PAM constellation with itself. Let $\mathbb{R}$ denotes the set of real numbers, using the optimization given by (3), finding the optimum nonuniform decomposable QAM constellations can be expressed as

$$
\begin{gathered}
\underset{\substack{\min _{i}^{(1)}, s_{i}^{(2} \\
\forall i \in\{1, \ldots, \sqrt{M}\}}}{\operatorname{minimize}} \sum_{i=1}^{\sqrt{M}} \sum_{j=i+1}^{\sqrt{M}} \frac{2 C}{\left(s_{i}^{(1)}-s_{j}^{(1)}\right)^{2}\left(s_{i}^{(2)}-s_{j}^{(2)}\right)^{2}} \\
\text { subject to } \frac{1}{\sqrt{M}} \sum_{i=1}^{\sqrt{M}}\left(s_{i}^{(t)}\right)^{2} \leq 1 \quad \forall t \\
s_{i}^{(1)}, s_{i}^{(2)} \in \mathbb{R} \quad \forall i .
\end{gathered}
$$

\footnotetext{
${ }^{2}$ This bound is derived assuming that the source symbols are equally likely; however, one can also derive a similar bound if the source symbols are not equally likely using the approach explained in [14].

${ }^{3}$ One can get a tighter bound on the pairwise error probability than that given in [6, eq. (6)] by using the $Q$-function expression given in [15, eq. (9)] instead of the Chernoff bound and get a smaller constant $\tilde{C}=(3 / 16) C$. However, since $C$ is a constant, it is irrelevant in the optimization. While the union bound is expected to be conservative, it is applicable to all possible CoRe schemes.
} 
Optimization (4) is not a convex optimization problem since the objective function is not convex (although the power constraints are convex). Nevertheless, we can partition the feasible set into a finite number of mutually exclusive convex sets in which the objective function is always convex. Partitioning is done using binary variables $o_{i j}^{(t)} \in\{0,1\}$, which relate pairs of symbols $s_{i}^{(t)}$ and $s_{j}^{(t)}$ for any $i, j \in\{1, \ldots, \sqrt{M}\}$ and a fixed $t$. We define these binary variables such that $o_{i j}^{(t)}=1$ implies $s_{i}^{(t)}>s_{j}^{(t)}$ and $o_{i j}^{(t)}=0$ implies $s_{i}^{(t)}<s_{j}^{(t)}$. Each fixed set of binary variables $\left\{o_{i j}^{(t)}: i, j \in\{1,2, \ldots, \sqrt{M}\}, t \in\right.$ $\{1,2\}\}$ corresponds to one of the mutually exclusive convex sets. The following proposition shows that the objective function is convex in each of these convex sets.

Proposition 1: The objective function in (4) is a convex function on a convex set $\Psi$ if, for any set of fixed binary variables $\left\{o_{i j}^{(t)}: i, j \in\right.$ $\{1, \ldots, \sqrt{M}\}, t \in\{1,2\}\}, \Psi$ is given by

$$
\begin{gathered}
\Psi=\left\{s_{1}^{(1)}, \ldots, s_{\sqrt{M}}^{(1)}, s_{1}^{(2)}, \ldots, s_{\sqrt{M}}^{(2)}: s_{i}^{(t)} \in \mathbb{R},\right. \\
-\left(1-o_{i j}^{(t)}\right) \mathbb{L}<s_{i}^{(t)}-s_{j}^{(t)}<o_{i j}^{(t)} \mathbb{L}, \\
o_{i j}^{(t)}=1-o_{j i}^{(t)}, o_{i j}^{(t)} \in\{0,1\}, \\
\forall i, j \in\{1, \ldots, \sqrt{M}\}, \forall t \in\{1,2\}\}
\end{gathered}
$$

where $\mathbb{L}$ is a large positive number

Proof: We start by the writing the objective function as

$$
f_{0}\left(s_{1}^{(1)}, \ldots, s_{\sqrt{M}}^{(1)}, s_{1}^{(2)}, \ldots, s_{\sqrt{M}}^{(2)}\right)=2 C \sum_{i=1}^{\sqrt{M}} \sum_{j=i+1}^{\sqrt{M}} f_{i j}
$$

where

$$
f_{i j}\left(s_{i}^{(1)}, s_{i}^{(2)}, s_{j}^{(1)}, s_{j}^{(2)}\right)=\left(s_{i}^{(1)}-s_{j}^{(1)}\right)^{-2} \times\left(s_{i}^{(2)}-s_{j}^{(2)}\right)^{-2} .
$$

Since convexity is preserved over addition [16, p. 79], the proof is reduced to proving that $f_{i j}(\cdot)$ is convex $\forall i, j \in\{1, \ldots, \sqrt{M}\}$. Taking the logarithm of $f_{i j}(\cdot)$, we get

$$
\begin{aligned}
\log f_{i j}\left(s_{i}^{(1)}, s_{i}^{(2)}, s_{j}^{(1)}, s_{j}^{(2)}\right)= & -\log \left(s_{i}^{(1)}-s_{j}^{(1)}\right)^{2} \\
& -\log \left(s_{i}^{(2)}-s_{j}^{(2)}\right)^{2} .
\end{aligned}
$$

Since $\frac{d^{2}\left(-\log \left(x^{2}\right)\right)}{d x^{2}}=\left(2 / x^{2}\right)>0, \quad-\log \left(x^{2}\right)$ is convex on $(0,+\infty)$ and convex on $(-\infty, 0)$. However, $-\log \left(x^{2}\right)$ is neither convex on $\mathbb{R}$, because it has singularity at $x=0$, nor convex on the set $\{x: x \neq 0\}$, because it is not a convex set. It follows that $-\log \left(s_{i}^{(t)}-\right.$ $\left.s_{j}^{(t)}\right)^{2}$ is convex on $\left\{s_{i}^{(t)}, s_{j}^{(t)}: s_{i}^{(t)}>s_{j}^{(t)}\right\}$ and convex on $\left\{s_{i}^{(t)}, s_{j}^{(t)}\right.$ : $\left.s_{i}^{(t)}<s_{j}^{(t)}\right\}$, for $t \in\{1,2\}$, because it is a composition of the convex function $-\log \left(x^{2}\right)$ with an affine function $\left(s_{i}^{(t)}-s_{j}^{(t)}\right)$. Equivalently, each term in the $\log f_{i j}$ 's addition is convex on $\left\{s_{i}^{(t)}, s_{j}^{(t)}: s_{i}^{(t)}, s_{j}^{(t)} \in\right.$ $\left.\mathbb{R},-\left(1-o_{i j}^{(t)}\right) \mathbb{L}<s_{i}^{(t)}-s_{j}^{(t)}<o_{i j}^{(t)} \mathbb{L}, o_{i j}^{(t)}=1-o_{j i}^{(t)}\right\}$. To see the equivalence, consider the following cases:

$$
\text { Case I: if } \begin{aligned}
o_{i j}^{(t)}=1 & \Rightarrow 0<s_{i}^{(t)}-s_{j}^{(t)}<\mathbb{L} \\
& \Rightarrow s_{i}^{(t)}>s_{j}^{(t)} .
\end{aligned}
$$

Case II: if $o_{i j}^{(t)}=0 \Rightarrow-\mathbb{L}<s_{i}^{(t)}-s_{j}^{(t)}<0$

$$
\Rightarrow s_{i}^{(t)}<s_{j}^{(t)} .
$$

Note that $o_{i j}^{(t)}=1-o_{j i}^{(t)}$ since $\log f_{i j}$ is not convex on the set $\left\{s_{i}^{(t)}, s_{j}^{(t)}: s_{i}^{(t)}, s_{j}^{(t)} \in \mathbb{R}, s_{i}^{(t)} \neq s_{j}^{(t)}\right\}$.

Being the sum of convex functions, $\log f_{i j}(\cdot)$ is convex. Hence, $f_{i j}(\cdot)$ is log-convex. Since $f_{i j}(\cdot)$ is log-convex, then it is also convex $[16, \mathrm{p} .104]$ if the conditions of the proposition are met.

Using Proposition 1 in (4), we pose the following $(0,1)$ mixedinteger programming problem:

$$
\begin{array}{rlr}
\underset{\substack{s_{i}^{(1)}, s_{i}^{(2)} \\
\forall i \in\{1, \ldots, \sqrt{M}\}}}{\operatorname{minimize}} \sum_{i=1}^{\sqrt{M}} \sum_{j=i+1}^{\sqrt{M}} \frac{2 C}{\left(s_{i}^{(1)}-s_{j}^{(1)}\right)^{2}\left(s_{i}^{(2)}-s_{j}^{(2)}\right)^{2}} \\
\text { subject to } & \frac{1}{\sqrt{M}} \sum_{i=1}^{\sqrt{M}}\left(s_{i}^{(t)}\right)^{2} \leq 1, & \forall t \\
& s_{i}^{(t)}-s_{j}^{(t)} \leq o_{i j}^{(t)} \mathbb{L}, & \forall i, j, t \\
& s_{j}^{(t)}-s_{i}^{(t)} \leq\left(1-o_{i j}^{(t)}\right) \mathbb{L}, & \forall i, j, t \\
& o_{i j}^{(t)}=1-o_{j i}^{(t)}, o_{i j}^{(t)} \in\{0,1\}, & \forall i, j, t
\end{array}
$$

where we introduced binary variables $o_{i j}^{(t)}$ to partition the feasible set according to the proposition. We note that the objective function prevents the equalities in partitioning constraints $(5 \mathrm{c})$ from being active. Thus, the constraints in the optimization are strictly true for optimal solutions, consistent with the proposition. In $(5 \mathrm{c}), \mathbb{L}$ can be set to any positive number greater than $2 M^{1 / 4}$ since $s_{i}^{(t)}-s_{j}^{(t)}<2 M^{1 / 4}$, $\forall i, j \in\{1, \ldots, \sqrt{M}\}, \forall t \in\{1,2\}$ to satisfy power constraints (5b).

The new partitioning constraints given by $(5 \mathrm{c})$ separate the optimization into multiple optimizations defined on mutually exclusive convex sets. Each of these optimization problem is defined by one set of fixed $o_{i j}^{(t)}$ variables. Taking the proposition into consideration, each of these optimization problems is convex and can be efficiently solved. Therefore, a convex optimizer is used to solve each of the convex optimization problems. Each solution is a local minimum of the original optimization problem. Then, we find the global minimum by taking the minimum of these local minimum points.

We found several computational shortcuts that are based on removing vast portions of unneeded space of all possible binary variables. Due to space limitations, the shortcuts are not described in the paper. Nevertheless, we discuss the number of total convex optimizations to give an idea of the time required to obtain one optimal set of constellations.

With all of our optimizations, we need to solve $\sqrt{M}$ ! optimization problems. For example, finding the optimum 4-PAM constellations (in-phase component of 16-QAM constellation) requires solving 24 problems, and finding the optimum 8-PAM constellations (in-phase component of 64-QAM constellation) requires solving 40320 problems. However, with the extremely efficient convex optimization algorithms, each convex optimization problem is solved on the order of milliseconds on a desktop computer. Since the problem is an "offline" optimization where the solution needs to be found only once, we found this optimization technique sufficient to solve the problem optimally.

\section{B. Nondecomposable Constellations}

For decomposable QAM constellations, the optimization problem was significantly simplified by designing optimal $\sqrt{M}$-PAM real constellations. However, this simplification does not hold for nondecomposable constellations, and thus, optimizing over complex constellations is inevitable. 
We start by reformulating the optimization given by (3) in terms of real numbers as

$$
\underset{\substack{x_{i}^{(1)}, y_{i}^{(1)}, x_{i}^{(2)}, y_{i}^{(2)} \\ \forall i \in\{1, \ldots, M\}}}{\operatorname{minimize}} \sum_{i=1}^{M} \sum_{j=i+1}^{M} \frac{2 C}{\prod_{t=1}^{2}\left[\left(x_{i}^{(t)}-x_{j}^{(t)}\right)^{2}+\left(y_{i}^{(t)}-y_{j}^{(t)}\right)^{2}\right]}
$$

$$
\begin{array}{ccc}
\text { subject to } & \frac{1}{M} \sum_{i=1}^{M}\left(\left(x_{i}^{(t)}\right)^{2}+\left(y_{i}^{(t)}\right)^{2}\right) \leq 1, \quad \forall t \\
x_{i}^{(1)}, y_{i}^{(1)}, x_{i}^{(2)}, y_{i}^{(2)} \in \mathbb{R}, & \forall i \quad \text { (6b) }
\end{array}
$$

where constraints (6b) and (6c) are obtained by direct substitution.

We note that objective function (6a) is differentiable and that the constraints are convex. However, the objective function itself is not convex (as we proved in [2]), precluding the possibility of finding a globally minimum solution with a standard solver. Nevertheless, we devise a simple heuristic that allows us to find local minima and provides very good solutions.

Although the objective function is not convex, we can still use an off-the-shelf solver to find local minima due to its differentiability. In this paper, we use the "fmincon" solver, which is part of the MATLAB optimization toolbox. In general, these solvers use gradientbased methods to get a local minimum. The local minimum found by the solver depends on the initial starting point; thus, a good starting point is essential to obtain good local minima. We use the optimum decomposable constellations found in Section IV-A as the starting point for the solver. Although the local minimum found using this starting point is not a global minimum, we are assured that it will perform at least as well as the optimum decomposable constellations. Indeed, simulations show that the constellations obtained this way outperform the optimum decomposable constellations.

\section{Simulation Results}

The optimized nonuniform CoRe schemes, which were explained in Section IV, can be applied to the generic system model explained in Section III. Here, we first explain one possible relaying protocol in Section V-A, which fits the generic system model. Then, we present simulation results in Section V-B.

\section{A. Simulation Setup}

The BS transmits a packet of $L$ bits in the first slot to the RS. Due to the broadcast nature of the wireless channel, the UT receives the packet from the BS. The RS fully decodes the packet and checks to determine if the packet is correctly decoded with the help of a cyclic redundancy check (CRC) code. ${ }^{4}$ If the packet is successfully decoded, the RS retransmits the packet to the UT in the second time slot. Otherwise, the RS refrains from retransmission and sends a 1-bit negative acknowledgment to the BS, indicating that it failed to decode the packet correctly, and the BS will use the second time slot to send the packet again to the UT. Finally, the UT utilizes the two transmissions to decode the packet in a symbol-by-symbol manner. With this protocol, detection errors made by the RS are not propagated to the UT.

Since the RS is fixed and can be installed at a strategic location where line-of-sight transmissions exist in most cases, the channel

\footnotetext{
${ }^{4}$ Using CRC does not imply an increase in the overhead since all the current and presumably future standards employ CRC anyway as a means to check the integrity of the received packets.
}

between the $\mathrm{BS}$ and the RS is modeled as a Rician random variables with a $K$-factor of $10 \mathrm{~dB}$. On the other hand, the channel between the BS and the UT and that between the RS and the UT are modeled as independent Rayleigh random variables due to the nonline-ofsight transmission from the BS and the RS to the UT. The average SNRs in BS-RS, RS-UT, and BS-UT links are denoted by $\bar{\gamma}^{\text {(BS-RS), }}$ $\bar{\gamma}^{(\mathrm{RS}-\mathrm{UT})}$, and $\bar{\gamma}^{(\mathrm{BS}-\mathrm{UT})}$, respectively.

\section{B. Results}

We use the algorithms described in Sections IV-A and IV-B to find 16-QAM and 64-QAM nonuniform decomposable and nonuniform nondecomposable constellations. We also obtain an optimized uniform CoRe scheme by the Cartesian product of optimum uniform PAM constellations found using the method outlined in [6].

Table I shows the optimized uniform, the optimized nonuniform decomposable, and the locally optimized nonuniform nondecomposable CoRe schemes. For the case of 16-QAM, the difference between the optimized nonuniform decomposable scheme and the optimum uniform scheme is small. Thus, we expect that the gain achieved by the optimized nonuniform decomposable scheme over the optimum uniform scheme to be small as well. On the other hand, the optimized nonuniform nondecomposable constellations are very different from the optimized uniform constellations, which suggests that they have the potential to achieve higher gains than their decomposable counterparts. For the case of 64-QAM, the signal points for both the optimized nonuniform decomposable and nondecomposable schemes are very different from the optimized uniform scheme. Therefore, one would expect higher performance gains than in the case of 16-QAM.

The optimized nonuniform decomposable and nondecomposable CoRe schemes are plotted in Fig. 3 for the case of 16-QAM. While the nonuniform decomposable constellation points form an irregularly spaced grid, the nonuniform nondecomposable constellation points do not form a grid, since by definition, these constellations are not a Cartesian product of two real constellations.

We simulate the performance of different CoRe schemes for the protocol described in Section V-A. We assume the size of the packet to be 188 bytes (video packet). We present the $\mathrm{SER}^{5}$ comparison curves for $\bar{\gamma}^{(\mathrm{BS}-\mathrm{RS})}=\bar{\gamma}^{(\mathrm{BS}-\mathrm{UT})}=\bar{\gamma}^{(\mathrm{RS}-\mathrm{UT})}($ symmetric case $), \bar{\gamma}^{(\mathrm{BS}-\mathrm{RS})}=$ $\bar{\gamma}^{(\mathrm{RS}-\mathrm{UT})}+15 \mathrm{~dB}$, and $\bar{\gamma}^{(\mathrm{BS}-\mathrm{UT})}=\bar{\gamma}^{(\mathrm{RS}-\mathrm{UT})}-15 \mathrm{~dB}$ (the UT is closer to the RS than the BS). We also observed similar gains for different packet sizes and different average SNR values.

Fig. 4(a) shows the SER performance of 16-QAM for different CoRe schemes. It includes the SER of the conventional scheme ("conventional"), where the same constellation is used by both the BS and the RS, the optimized uniform CoRe scheme ("optimized uniform"), the optimized nonuniform decomposable CoRe scheme ("optimized nonuniform decomposable"), and the locally optimized nonuniform nondecomposable CoRe scheme ("optimized nonuniform nondecomposable"). In all of these CoRe schemes, optimality is defined as minimizing the union bound on the uncoded SER. We have found that the optimized uniform scheme outperforms all other uniform CoRe schemes described in the literature; thus, we only show its results. The optimized nonuniform decomposable scheme yields a gain of $2.8 \mathrm{~dB}$ over the conventional scheme and a modest gain of $0.2 \mathrm{~dB}$ over the optimum uniform scheme, at SER $=10^{-3}$. We note, however, that this gain is achieved with no penalty on complexity. On the other hand, the optimized nonuniform nondecomposable scheme yields a 2.8-dB gain over the conventional scheme and a $0.5-\mathrm{dB}$ gain over the optimum uniform CoRe scheme, at SER $=10^{-3}$, at the cost of increased decoding complexity.

\footnotetext{
${ }^{5}$ Packet error ratio results can be derived from SER results.
} 
TABLE I

OPTIMIZED $M$-QAM CoRe CONSTELLATIONS

\begin{tabular}{|c|c|c|}
\hline$M$ & Slot & Constellation $\left\{s_{1}^{(t)}, \ldots, s_{M}^{(t)}\right\}$ \\
\hline & & Optimized uniform \\
\hline \multirow{2}{*}{16} & $t=1$ & $\begin{array}{c}\{-3-3 j,-3-1 j,-3+1 j,-3+3 j,-1-3 j,-1-1 j,-1+1 j,-1+3 j \\
1-3 j, 1-1 j, 1+1 j, 1+3 j, 3-3 j, 3-1 j, 3+1 j, 3+3 j\} \times 0.3162\end{array}$ \\
\hline & $t=2$ & $\begin{array}{c}\{-1-1 j,-1+3 j,-1-3 j,-1+1 j, 3-1 j, 3+3 j, 3-3 j, 3+1 j \\
-3-1 j,-3+3 j,-3-3 j,-3+1 j, 1-1 j, 1+3 j, 1-3 j, 1+1 j\} \times 0.3162 \\
\end{array}$ \\
\hline \multirow{2}{*}{64} & $t=1$ & $\begin{array}{l}\{-7-7 j,-7-5 j,-7-3 j,-7-1 j,-7+1 j,-7+3 j,-7+5 j,-7+7 j,-5-7 j,-5-5 j,-5-3 j,-5-1 j \\
-5+1 j,-5+3 j,-5+5 j,-5+7 j,-3-7 j,-3-5 j,-3-3 j,-3-1 j,-3+1 j,-3+3 j,-3+5 j,-3+7 j \\
-1-7 j,-1-5 j,-1-3 j,-1-1 j,-1+1 j,-1+3 j,-1+5 j,-1+7 j, 1-7 j, 1-5 j, 1-3 j, 1-1 j, 1+1 j \\
\quad 1+3 j, 1+5 j, 1+7 j, 3-7 j, 3-5 j, 3-3 j, 3-1 j, 3+1 j, 3+3 j, 3+5 j, 3+7 j, 5-7 j, 5-5 j \\
5-3 j, 5-1 j, 5+1 j, 5+3 j, 5+5 j, 5+7 j, 7-7 j, 7-5 j, 7-3 j, 7-1 j, 7+1 j, 7+3 j, 7+5 j, 7+7 j\} \times 0.1543\end{array}$ \\
\hline & $t=2$ & $\begin{array}{c}\{-1-1 j,-1+5 j,-1-5 j,-1+1 j,-1+7 j,-1-7 j,-1+3 j,-1-3,5-1 j, 5+5 j, 5-5 j, 5+1 j, 5+7 j, \\
5-7 j, 5+3 j, 5-3 j,-5-1 j,-5+5 j,-5-5 j,-5+1 j,-5+7 j,-5-7 j,-5+3 j,-5-3 j, 1-1 j, \\
1+5 j, 1-5 j, 1+1 j, 1+7 j, 1-7 j, 1+3 j, 1-3 j, 7-1 j, 7+5 j, 7-5 j, 7+1 j, 7+7 j, \\
7-7 j, 7+3 j, 7-3 j,-7-1 j,-7+5 j,-7-5 j,-7+1 j,-7+7 j,-7-7 j,-7+3 j,-7-3 j, 3-1 j, 3+5 j, \\
3-5 j, 3+1 j, 3+7 j, 3-7 j, 3+3 j, 3-3 j,-3-1 j,-3+5 j,-3-5 j,-3+1 j,-3+7 j,-3-7 j,-3+3 j,-3-3 j\} \times 0.1543 \\
\end{array}$ \\
\hline \multicolumn{3}{|r|}{ Optimized nonuniform decomposable } \\
\hline \multirow{2}{*}{16} & $t=1$ & $\begin{array}{c}\{-3.05-3.05 j,-3.05-0.84 j,-3.05+0.84 j,-3.05+3.05 j,-0.84-3.05 j,-0.84-0.84 j,-0.84+0.84 j,-0.84+3.05 j \\
0.84-3.05 j, 0.84-0.84 j, 0.84+0.84 j, 0.84+3.05 j, 3.05-3.05 j, 3.05-0.84 j, 3.05+0.84 j, 3.05+3.05 j\} \times 0.3162 \\
\end{array}$ \\
\hline & $t=2$ & $\begin{array}{c}\{-0.84-0.84 j,-0.84+3.05 j,-0.84-3.05 j,-0.84+0.84 j, 3.05-0.84 j, 3.05+3.05 j, 3.05-3.05 j, 3.05+0.84 j \\
-3.05-0.84 j,-3.05+3.05 j,-3.05-3.05 j,-3.05+0.84 j, 0.84-0.84 j, 0.84+3.05 j, 0.84-3.05 j, 0.84+0.84 j\} \times 0.3162 \\
\end{array}$ \\
\hline \multirow{2}{*}{64} & $t=1$ & $\begin{array}{c}\{-7.20-7.20 j,-7.20-4.57 j,-7.20-2.63 j,-7.20-1.29 j,-7.20+0.55 j,-7.20+2.96 j,-7.20+4.74 j,-7.20+7.43 j \\
-4.57-7.20 j,-4.57-4.57 j,-4.57-2.63 j,-4.57-1.29 j,-4.57+0.55 j,-4.57+2.96 j,-4.57+4.74 j,-4.57+7.43 j \\
-2.63-7.20 j,-2.63-4.57 j,-2.63-2.63 j,-2.63-1.29 j,-2.63+0.55 j,-2.63+2.96 j,-2.63+4.74 j,-2.63+7.43 j \\
-1.29-7.20 j,-1.29-4.57 j,-1.29-2.63 j,-1.29-1.29 j,-1.29+0.55 j,-1.29+2.96 j,-1.29+4.74 j,-1.29+7.43 j \\
\quad 0.55-7.20 j, 0.55-4.57 j, 0.55-2.63 j, 0.55-1.29 j, 0.55+0.55 j, 0.55+2.96 j, 0.55+4.74 j, 0.55+7.43 j \\
\quad 2.96-7.20 j, 2.96-4.57 j, 2.96-2.63 j, 2.96-1.29 j, 2.96+0.55 j, 2.96+2.96 j, 2.96+4.74 j, 2.96+7.43 j \\
\quad 4.74-7.20 j, 4.74-4.57 j, 4.74-2.63 j, 4.74-1.29 j, 4.74+0.55 j, 4.74+2.96 j, 4.74+4.74 j, 4.74+7.43 j \\
\quad 7.43-7.20 j, 7.43-4.57 j, 7.43-2.63 j, 7.43-1.29 j, 7.43+0.55 j, 7.43+2.96 j, 7.43+4.74 j, 7.43+7.43 j\} \times 0.1543\end{array}$ \\
\hline & $t=2$ & $\begin{array}{c}\{-2.97-2.97 j,-2.97+2.54 j,-2.97-7 . j,-2.97+7.61 j,-2.97-1.08 j,-2.97+4.64 j,-2.97-4.71 j,-2.97+0.97 j \\
2.54-2.97 j, 2.54+2.54 j, 2.54-7 . j, 2.54+7.61 j, 2.54-1.08 j, 2.54+4.64 j, 2.54-4.71 j, 2.54+0.97 j \\
-7.00-2.97 j,-7.00+2.54 j,-7.00-7 . j,-7.00+7.61 j,-7.00-1.08 j,-7.00+4.64 j,-7.00-4.71 j,-7.00+0.97 j \\
\quad 7.61-2.97 j, 7.61+2.54 j, 7.61-7 . j, 7.61+7.61 j, 7.61-1.08 j, 7.61+4.64 j, 7.61-4.71 j, 7.61+0.97 j \\
-1.08-2.97 j,-1.08+2.54 j,-1.08-7 . j,-1.08+7.61 j,-1.08-1.08 j,-1.08+4.64 j,-1.08-4.71 j,-1.08+0.97 j \\
\quad 4.64-2.97 j, 4.64+2.54 j, 4.64-7 . j, 4.64+7.61 j, 4.64-1.08 j, 4.64+4.64 j, 4.64-4.71 j, 4.64+0.97 j \\
-4.71-2.97 j,-4.71+2.54 j,-4.71-7 . j,-4.71+7.61 j,-4.71-1.08 j,-4.71+4.64 j,-4.71-4.71 j,-4.71+0.97 j \\
0.97-2.97 j, 0.97+2.54 j, 0.97-7 . j, 0.97+7.61 j, 0.97-1.08 j, 0.97+4.64 j, 0.97-4.71 j, 0.97+0.97 j\} \times 0.1543\end{array}$ \\
\hline \multicolumn{3}{|r|}{$\begin{array}{l}\text { Locally optimized nonuniform nondecomposable } \\
\end{array}$} \\
\hline \multirow{2}{*}{16} & $t=1$ & $\begin{array}{l}\{-1.87-3.78 j,-3.48+1.13 j,-1.23+1.77 j,-2.18+3.63 j, 2.56-1.90 j, 0.19-2.40 j,-3.61-1.10 j,-1.04+0.23 j \\
1.66-3.72 j, 1.73+0.15 j,-1.67-1.25 j, 0.68+3.86 j, 4.05+0.19 j, 2.95+2.42 j, 0.44-0.93 j, 0.83+1.71 j\} \times 0.3162\end{array}$ \\
\hline & $t=2$ & $\begin{array}{c}\{0.09+0.46 j,-0.95+2.34 j,-2.47-3.02 j, 1.60-0.10 j, 3.26+0.09 j, 0.34+4.05 j, 1.34-2.17 j, 3.35+2.46 j \\
-2.18-1.23 j,-2.97+2.67 j,-4.05+0.13 j,-1.81+0.33 j,-0.17-1.49 j, 1.11+2.08 j, 0.15-4.20 j, 3.37-2.41 j\} \times 0.3162\end{array}$ \\
\hline \multirow{2}{*}{64} & $t=1$ & $\begin{array}{c}\{-7.44-6.55 j,-7.08-3.50 j,-5.83-1.51 j,-8.91-1.46 j,-8.73+1.54 j,-5.23+1.31 j,-2.67+1.31 j,-8.15+4.38 j \\
-2.77-8.43 j,-4.50-6.10 j,-4.98-3.89 j,-4.30-2.16 j,-6.82+0.17 j,-3.49+3.17 j,-5.84+3.30 j,-5.79+7.35 j \\
-2.36-5.97 j,-2.94-3.81 j,-0.32-0.19 j,-0.94+2.58 j,-3.59-0.49 j, 2.50+2.89 j, 0+4.33 j,-4.34+5.04 j \\
0.09-9.13 j,-1 .-4.48 j, 1.30-0.87 j,-1.03+0.90 j,-2.40-1.16 j, 1.07+3.20 j,-1.74+4.07 j,-3.13+6.41 j \\
2.09-7.67 j,-0.12-6.58 j,-1.53-2.99 j,-0.82-1.67 j, 1.09+1.21 j, 0.95+5.92 j,-1.01+6.57 j,-2.24+8.77 j \\
\quad 1.46-4.90 j, 2.81-2.34 j, 3.92+2.28 j, 3.04+0.78 j, 5.46+0.66 j, 4.13+4.12 j, 2.75+5.59 j, 0.66+8.78 j \\
\quad 0.75-2.78 j, 3.12-5.16 j, 3.59-0.61 j, 4.83-1.91 j, 6.60-0.82 j, 6.05+2.62 j, 5.55+4.84 j, 3.34+8.34 j \\
4.74-8.37 j, 7.72-5.19 j, 5.03-3.67 j, 4.93-5.65 j, 8.41-2.50 j, 8.66+0.24 j, 8.64+3.02 j, 6.74+6.84 j\} \times 0.1543\end{array}$ \\
\hline & $t=2$ & $\begin{array}{c}\{-0.85-1.29 j,-4.10+1.66 j,-3.64-6.41 j, 0.33+5.36 j,-3.75-2.56 j,-3.47+5.06 j,-0.79-3.67 j,-0.17+2.20 j, \\
\quad 1.55-3.50 j, 2.26+2.55 j, 4.30-5.60 j, 3.85+7.38 j, 4.61-1 . j, 2.58+4.51 j, 2.05-6.32 j, 1.57-1.63 j, \\
-6.02-3.64 j,-6.71+4.65 j,-6.94-6.63 j,-6.34+7.21 j,-8.86-0.43 j,-8.85+2.59 j,-8.09-3.35 j,-5.82+0.25 j, \\
\quad 5.34+0.77 j, 7.89+3.58 j, 6.14-7.46 j, 7.23+6.67 j, 8.78-2.06 j, 9.25+1.10 j, 6.96-4.63 j, 6.34+2.28 j \\
-2.59-4.08 j,-1.59+3.97 j,-0.10-8.67 j,-1.48+8.84 j,-0.23+0.30 j,-1.11+6.33 j,-1.40-6.43 j,-2.48+2.42 j, \\
\quad 5.40-3.23 j, 4.27+2.75 j, 2.49-8.40 j, 1.84+9.05 j, 6.71-0.79 j, 4.86+5.19 j, 3.22-3.97 j, 3.52+0.55 j \\
-3.80-0.62 j,-6.74+1.91 j,-3.39-8.50 j,-3.75+7 . j,-6.23-1.64 j,-4.43+3.38 j,-4.35-4.63 j,-2.36-1.34 j, \\
1.66+0.01 j,-2.17+0.92 j, 0.47-5.54 j, 1.87+6.31 j, 3.26-1.85 j, 0.48+3.86 j, 0.18-2.40 j, 1.34+1.63 j\} \times 0.1543\end{array}$ \\
\hline
\end{tabular}




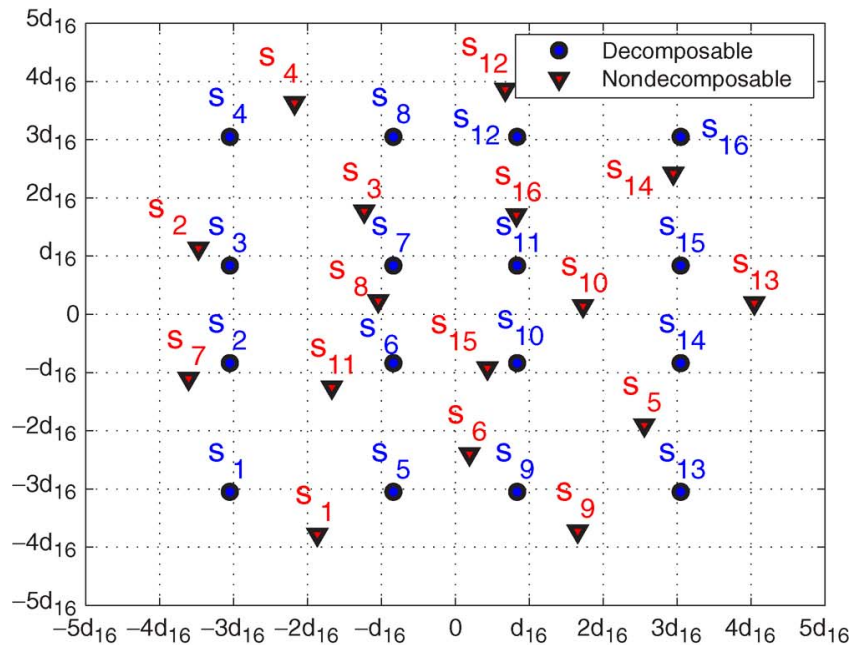

(a)

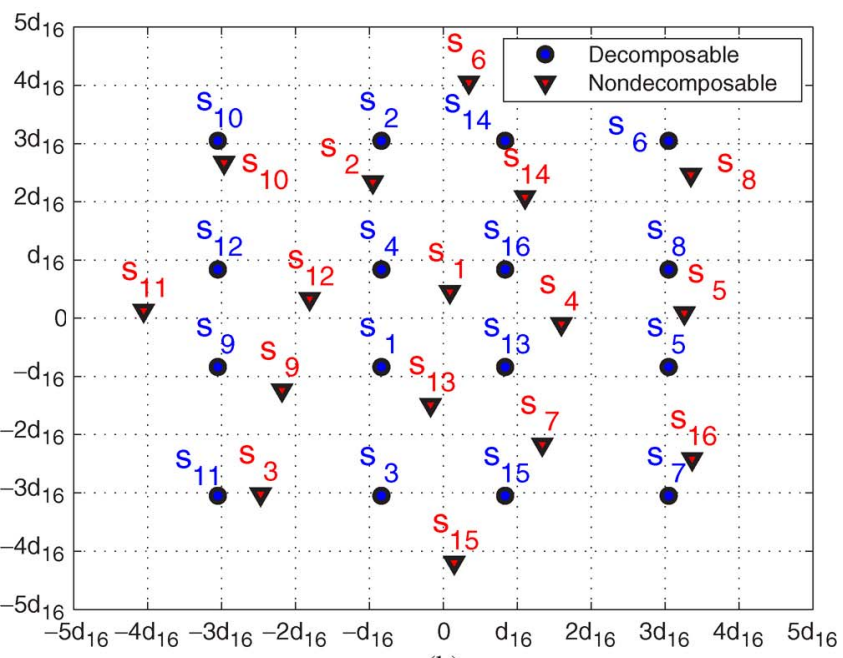

(b)

Fig. 3. Optimized nonuniform CoRe schemes for the case of 16-QAM (a) Signal constellations used at the BS. (b) Signal constellations used at the RS.

Fig. 4(b) shows the SER performance results for the case of 64-QAM, for the same CoRe schemes. The optimized nonuniform decomposable scheme yields a gain of $4.3 \mathrm{~dB}$ over the conventional scheme and a gain of $0.6 \mathrm{~dB}$ over the optimized uniform scheme, at $\mathrm{SER}=10^{-3}$. This gain comes at no extra complexity. On the other hand, the optimized nonuniform nondecomposable scheme yields a 4.7- $\mathrm{dB}$ gain over the conventional scheme and a $0.9-\mathrm{dB}$ gain over the optimum uniform CoRe scheme, at SER $=10^{-3}$, at the cost of increased decoding complexity.

From the simulation results, the following design guidelines can be made. For scenarios where decoding complexity is less of an issue, nonuniform nondecomposable CoRe schemes should be used for both cases of 16- and 64-QAM. On the other hand, for scenarios where decoding complexity should be kept at minimum, optimized uniform CoRe should be used for the case of 16-QAM, and optimized nonuniform decomposable CoRe should be used for the case of 64-QAM.

\section{CONCLUSION}

In the literature, CoRe is restricted to uniform constellations with regularly spaced signal points. In this paper, we have investi-

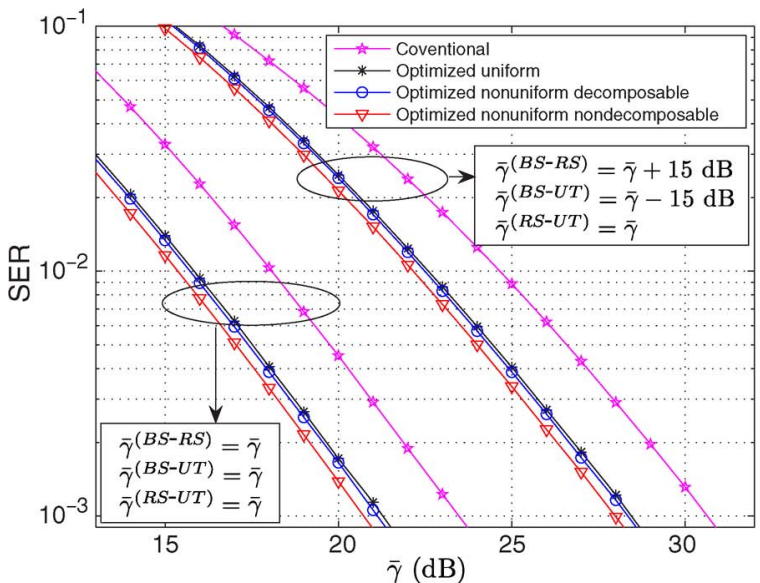

(a)

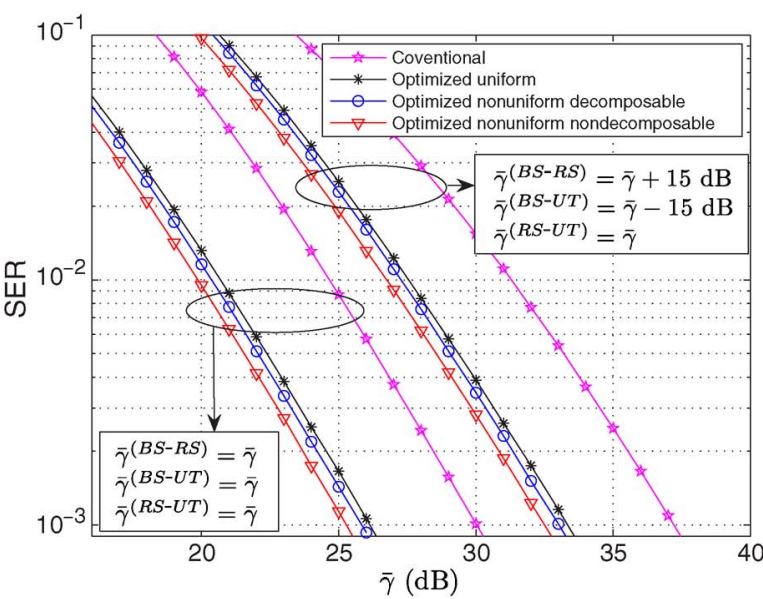

(b)

Fig. 4. SER performance results for different CoRe schemes. (a) 16-QAM. (b) 64-QAM

gated the problem of designing optimum nonuniform CoRe schemes. Nonuniform QAM constellations can be categorized as either decomposable or nondecomposable. Unlike nondecomposable QAM constellations, decomposable QAM constellations are generated from the Cartesian product of two PAM constellations.

Using convex analysis, we have shown that the problem of finding the minimum SER nonuniform decomposable CoRe can be cast as a series of convex optimization problems. Using this insight, we have obtained the optimum nonuniform decomposable 16-QAM and 64-QAM constellations that minimize the union bound on the uncoded SER.

Although the problem of finding the minimum SER nonuniform nondecomposable QAM constellation is not a convex optimization problem, we can still use a convex solver to find good locally optimum constellations. We take the optimum decomposable constellations as the starting point to the optimization so that the solver always finds constellations that are at least as good as the optimum decomposable constellations but with some increase in decoding complexity.

Considering the gain and decoding complexity of each scheme, the following design guidelines can be made. For scenarios where decoding complexity is less of an issue, nonuniform nondecomposable CoRe schemes should be used for both cases of 16-QAM and 64-QAM. On the other hand, for scenarios where decoding complexity should be kept at minimum, optimized uniform CoRe should be used for the case of 16-QAM, and optimized nonuniform decomposable CoRe should be used for the case of 64-QAM. 
There are a number of interesting open research problems related to nonuniform CoRe. Designing optimum nonuniform CoRe schemes for coded systems is the next natural step. For the cases of trellis-coded modulation systems, these problems may be tackled by combining the approach explained in this paper and those proposed in [17] and [18]. The use of nonuniform CoRe may also be fruitful in multiantenna systems.

\section{ACKNOWLEDGMENT}

The authors would like to thank Dr. I. Marsland from Carleton University and Dr. S. Loyka from the University of Ottawa for valuable discussions.

\section{REFERENCES}

[1] A. Bin Sediq, P. Djukic, H. Yanikomeroglu, and J. Zhang, "Near-optimal non-uniform constellation rearrangement for cooperative relaying," in Proc. 25th Queen's Biennial Symp. Commun., Kingston, ON, Canada, May 2010.

[2] A. Bin Sediq, P. Djukic, H. Yanikomeroglu, and J. Zhang, "Generalized constellation rearrangement in cooperative relaying," in Proc. 71st IEEE VTC-Spring, Taipei, Taiwan, May 2010, pp. 1-5.

[3] J. N. Laneman, D. Tse, and G. Wornell, "Cooperative diversity in wireless networks: Efficient protocols and outage behavior," IEEE Trans. Inf. Theory, vol. 50, no. 12, pp. 3062-3080, Dec. 2004.

[4] K. G. Seddik, A. Ibrahim, and K. Liu, "Trans-modulation in wireless relay networks," IEEE Commun. Lett., vol. 12, no. 3, pp. 170-172, Mar. 2008

[5] J. Yamazaki, T. Ikeda, M. Asa, and M. M. Mollah, "Performance of symbol combining with constellation change in multihop relay," in Proc. 66th IEEE VTC_Fall, Baltimore, MD, Oct. 2007, pp. 1694-1697.

[6] M. N. Khormuji and E. G. Larsson, "Improving collaborative transmit diversity by using constellation rearrangement," in Proc. IEEE WCNC, Hong Kong, Mar. 2007, pp. 803-807.

[7] H. Samra, Z. Ding, and P. M. Hahn, "Symbol mapping diversity design for multiple packet transmissions," IEEE Trans. Commun., vol. 53, no. 5, pp. 810-817, May 2005.

[8] C. Wengerter, A. V. Elbwart, and E. Seidel, "Constellation rearrangement: Enhancement for multilevel modulation formats and transmit diversity," Wireless Pers. Commun., vol. 29, no. 1/2, pp. 35-45, Apr. 2004.

[9] M. Gidlund and Y. Xu, "An improved ARQ schemes with application to multi-level modulation techniques," in Proc. IEEE Int. Symp. Commun. Inf. Technol., Sapporo, Japan, Oct. 2004, pp. 973-978.

[10] R. Otnes and T. Maseng, "Adaptive data rate using ARQ and nonuniform constellations," in Proc. 53rd IEEE VTC-Spring, Rhodes, Greece, May 2001, pp. 1211-1215.

[11] C. Wengerter, A. G. E. von Elbwart, E. Seidel, G. Velev, and M. Schmitt, "Advanced hybrid ARQ technique employing a signal constellation rearrangement," in Proc. 56th IEEE VTC - Fall, Vancouver, BC, Canada, Sep. 2002, pp. 2002-2006.

[12] M. Gidlund, "Performance of combined constellation rearrangement and space-time block coding scheme for multi-level modulation," in Proc. 18th IEEE PIMRC, Athens, Greece, Sep. 2007, pp. 1-5.

[13] H. Chen, Q. Chen, and P. Fan, "A modified HARQ scheme associated with non-uniform constellation," in Proc. 4th Int. Conf. Parallel Distrib. Comput., Appl. Technol., Baltimore, MD, Aug. 2003, pp. 447-451.

[14] H. Kuai, F. Alajaji, and G. Takahara, "Tight error bounds for nonuniform signaling over AWGN channels," IEEE Trans. Inf. Theory, vol. 46, no. 7 , pp. 2712-2718, Nov. 2000.

[15] J. Craig, "A new, simple and exact result for calculating the probability of error for two-dimensional signal constellations," in Proc. IEEE MILCOM, McLean, VA, Nov. 1991, pp. 571-575.

[16] S. Boyd and L. Vandenberghe, Convex Optimization. Cambridge, U.K.: Cambridge Univ. Press, 2004.

[17] J. Yu, Y. Li, H. Murata, and S. Yoshida, "Hybrid-ARQ scheme using different TCM for retransmission," IEEE Trans. Commun., vol. 48, no. 10, pp. 1609-1613, Oct. 2000.

[18] T. Koike, H. Murata, and S. Yoshida, "Hybrid ARQ scheme suitable for coded MIMO transmission," in Proc. IEEE Int. Conf. Commun., Paris, France, 2004, vol. 5, pp. 2919-2923.

\section{Hybridizing Biogeography-Based Optimization With Differential Evolution for Optimal Power Allocation in Wireless Sensor Networks}

\author{
Ilhem Boussaïd, Amitava Chatterjee, \\ Patrick Siarry, Senior Member, IEEE, and \\ Mohamed Ahmed-Nacer
}

\begin{abstract}
This paper studies the performance of a wireless sensor network (WSN) in the context of binary detection of a deterministic signal. This paper aims to develop a numerical solution for the optimal power allocation scheme via a variation of the biogeography-based optimization (BBO) algorithm, which is called the constrained BBO-DE algorithm. This new stochastic optimization algorithm is a hybridization of a very recently proposed stochastic optimization algorithm, i.e., the BBO algorithm, with another popular stochastic optimization algorithm called the differential evolution (DE) algorithm. The objective is to minimize the total power spent by the whole sensor network under a desired performance criterion, which is specified as the detection error probability. The proposed algorithm has been tested for several case studies, and its performances are compared with those of two constrained versions of the BBO and DE algorithms.
\end{abstract}

Index Terms-Biogeography-based optimization (BBO) algorithm, differential evolution (DE) algorithm, optimal power allocation, wireless sensor network (WSN).

\section{INTRODUCTION}

A wireless sensor network (WSN) is an infrastructure comprising spatially distributed sensor nodes with the abilities of sensing (measuring), computing, and communicating through the wireless channels. The development of WSNs was motivated by many application areas such as environment monitoring, health, security, and detection of remote parameters [1].

In a distributed detection system, every sensor node performs some preliminary processing of data and transmits a local decision to the fusion center. Based on the received data, the fusion center is responsible for the final decision making [2]. The distributed schemes offer the possibility for drastic reductions in communication requirements, at the expense of some performance degradation [3].

The key motivation of this paper is to optimize the performance of the system with respect to a desired performance criterion, which is specified as the detection error probability at the fusion center. The decision rule at the fusion center, along with the local sensor decision rules, needs to be jointly designed to optimize the specified performance criterion.

This paper aims at developing a numerical solution for optimal power scheduling in WSNs for correlated observations. Two constrained variants of the biogeography-based optimization (BBO) algorithm have been proposed to address this issue. They are known as $\mathrm{CBBO}$ with the conventional $\mathrm{BBO}-$ based operators and CBBO-DE, which incorporates the mutation procedure inherited from differential

Manuscript received July 23, 2010; revised January 24, 2011 and March 22, 2011; accepted April 18, 2011. Date of publication May 5, 2011; date of current version June 20,2011. The review of this paper was coordinated by $\mathrm{Dr}$. L. Li.

I. Boussaïd and M. Ahmed-Nacer are with the University of Science and Technology6 Houari Boumediene, 16111 Algiers, Algeria.

A. Chatterjee is with the Department of Electrical Engineering, Jadavpur University, Kolkata 700 032, India.

P. Siarry is with LiSSi (EA 3956), Université de Paris-Est Créteil Val de Marne, 94010 Créteil, France.

Digital Object Identifier 10.1109/TVT.2011.2151215 